\title{
Pairwise Error Probability of Space-Time Codes in Rician-Nakagami Channels
}

\author{
Murat Uysal, Member, IEEE
}

\begin{abstract}
In this letter, we investigate the shadowing effect on the performance of space-time codes, using the Rician-Nakagami channel model which has been recently introduced. Specifically, we derive an exact expression for the pairwise error probability (PEP) of space-time trellis codes over the Rician-Nakagami channel, which is in the form of a simple single finite-range integral. We also present numerical results how the PEP expressions for the Rician-Nakagami channel can be related to those for the classical Rician-lognormal channel based on the parameter transformation between these two models.
\end{abstract}

Index Terms-Nakagami model, pairwise error probability (PEP), shadowed Rician model, shadowing, space-time trellis coding.

\section{INTRODUCTION}

$\mathbf{S}$ INCE the introduction of space-time coding [1], a significant amount of work has been published on the design, decoding and applications of this new family of codes. Most of these results, however, are based on the assumption that the channel fading is either Rayleigh or Rician distributed, which are mostly used for the land mobile terrestrial (LMT) systems. As a consequence of the growing interest in land mobile satellite (LMS) systems, much effort is being devoted to the problem of modeling multipath fading and shadow fading in LMS channels. A comprehensive list of available LMS channel models can be found in [2]. Among the different viable approaches, Loo's model [3] is widely accepted and used extensively in the literature. This model assumes that the received signal envelope is described by a Rician model with lognormal shadowing on the line-of-sight (LOS) component ${ }^{1}$. Although the Rician-lognormal (R-L) model provides good match to experimental data, the assumption of lognormal shadowing results in complicated expressions for analytical error rate performance evaluation. Motivated by the inherent disadvantages of the R-L model, Abdi et al. [2] introduced a new simple LMS channel model, which is basically a Rician channel where the amplitude of LOS component is assumed to follow a Nakagami distribution. The proposed Rician-Nakagami $(\mathrm{R}-\mathrm{N})$ model is shown to

Manuscript received July 1, 2003. The associate editor coordinating the review of this manuscript and approving it for publication was Prof. K. Zhang. This paper was presented in part at the 41th Annual Allerton Conference on Communications, Control and Computing, Monticello, IL, October 2003.

The author is with the Department of Electrical and Computer Engineering, University of Waterloo, ON N2L 3G1Canada (e-mail: muysal@ ece.uwaterloo.ca).

Digital Object Identifier 10.1109/LCOMM.2004.823401

${ }^{1}$ Hereinafter, we will use the term "Rician-lognormal" to refer Loo's model. However, it should be noted that there are other LMS models, where the multipath fading and multiplicative shadow fading are described by Rician and lognormal distributions, respectively [2]. provide a similar fit to the experimental data as the $\mathrm{R}-\mathrm{L}$ model but with less computational complexity.

In this letter, we analyze the performance of space-time trellis codes over the $\mathrm{R}-\mathrm{N}$ channel via the derivation of a pairwise error probability (PEP) expression. PEP is the basic tool for the derivation of union bounds on the error rate performance of a coded communication system and gives valuable insights into the code design problem. In their pioneering work on space-time codes, Tarokh et al. [1] derived an upper bound on the PEP using the classical Chernoff bound over the Rayleigh and Rician fading channels. Exact PEP expressions for space-time codes were also derived using different approaches [4]-[7]. Recently, in [8], the author investigated the performance of space-time trellis codes over LMS channels assuming the R-L model. However, due to the nature of the lognormal assumption, the final expression for the PEP presented in [8] does not accept a simple form and is given in terms of an infinite summation, where truncation is possible for some practical values of channel parameters. Motivated by the simple transformation between the R-L and $\mathrm{R}-\mathrm{N}$ channel models, in this letter we re-derive the PEP for space-time coded systems assuming the Nakagami-distributed LOS component, expecting a much simpler solution.

\section{TRANSMISSION MODEL}

We consider a wireless communication system with $M$ transmit antennas and $N$ receive antennas. The binary data stream is first modulated and mapped to a sequence of complex modulation symbols. The modulated sequence is then fed to the space-time encoder and transmitted over the wireless channel. The receiver employs a maximum-likelihood decoder and has perfect channel state information (CSI). The received signal in the time interval $l$ at the $n$th receive antenna is given as

$$
r_{l, n}=\sum_{m=1}^{M} \alpha_{m, n} x_{m, l}+z_{l, n}
$$

where $x_{m, l}$ is a complex valued modulation symbol transmitted from the $m$ th $(m=1,2 \ldots M)$ transmit antenna at time $l$ and $z_{l, n}$ is the additive thermal noise term, the collection of which is modeled as independent samples of a zero-mean complex Gaussian random variable with variance $N_{0} / 2$ per dimension. The fading coefficient $\alpha_{m, n}$ represents the channel gain from transmit antenna $m$ to receive antenna $n$ and assumed to be constant over the duration of one frame, following a quasistatic channel assumption. The $\alpha_{m, n}$ 's are modeled as independent and identically distributed (i.i.d) samples of complex Gaussian random variables with mean $\mathrm{E}\left(\alpha_{m, n}\right)$ and variance $\operatorname{var}\left(\alpha_{m, n}\right)=\sigma_{\alpha}^{2}$ per dimension, leading to a Rician fading 
model. In our case, the LOS (corresponding to the nonzero mean) component is not constant but rather a Nakagamidistributed random variable following the probability density function given as

$$
p(\mu)=\frac{2 \eta^{\eta}}{\Gamma(\eta) \Lambda^{\eta}} \mu^{2 \eta-1} \exp \left(-\frac{\eta}{\Lambda} \mu^{2}\right), \quad \mu \geq 0
$$

where $\eta$ is the Nakagami parameter, $\Lambda=E\left(\mu^{2}\right)$ is the average power of the LOS component and $\Gamma($.$) is the gamma function.$ Contrary to the traditional Nakagami model where $\eta$ changes over the limited range of $\eta \geq 0.5$, here $\eta$ varies over the wider range of $\eta \geq 0$ [2].

\section{Derivation of Pairwise ERror Probability}

Let $\boldsymbol{x}_{l}=\left|x_{1, l}, x_{2, l}, \ldots, x_{M, l}\right|$ denote the code vector transmitted from $M$ transmit antennas in time interval $l$. For a frame length of $L$ time intervals, we denote the codeword matrix as $\boldsymbol{X}=\left|\boldsymbol{x}_{1}^{T}, \boldsymbol{x}_{2}^{T}, \ldots, \boldsymbol{x}_{L}^{T}\right|$ where $(.)^{T}$ represents transpose operation. The PEP represents the probability of choosing the code matrix $\hat{\boldsymbol{X}}$ when indeed $\boldsymbol{X}$ was transmitted. Under the assumption of perfect CSI, the conditional PEP is given by [1]

$$
P\left(\boldsymbol{X}, \hat{\boldsymbol{X}} \mid \alpha_{m}^{n}\right)=Q\left(\sqrt{\frac{E_{s}}{2 N_{0}} \sum_{n=1}^{N} \boldsymbol{\alpha}_{n} \mathbf{A} \boldsymbol{\alpha}_{n}^{H}}\right) .
$$

where $(.)^{H}$ represents conjugate transpose operation and $Q($. is the Gaussian $Q$-function. Here, $E_{s}$ represents the energy per symbol, $\boldsymbol{\alpha}_{n}=\left(\alpha_{1, n}, \alpha_{2, n}, \ldots, \alpha_{M, n}\right)$ is the channel vector and $\boldsymbol{A}=(\boldsymbol{X}-\hat{\boldsymbol{X}})(\boldsymbol{X}-\hat{\boldsymbol{X}})^{H}$. Following [1], we write

$$
\boldsymbol{\alpha}_{n} A \boldsymbol{\alpha}_{n}^{H} \stackrel{d}{=} \sum_{m=1}^{M} \lambda_{m}\left|\beta_{m, n}\right|^{2}
$$

where $\lambda_{m}$ are the eigenvalues of $A$ and $\beta_{m, n}=\sum_{k=1}^{M} \alpha_{k, n} v_{k, m}^{*}$ is the $m$ th element of the $M$-length vector $\beta_{n}=\boldsymbol{\alpha}_{n} \boldsymbol{V}^{H}$. Here, $\boldsymbol{V}$ is a unitary matrix such that $\boldsymbol{V} \boldsymbol{A} \boldsymbol{V}^{H}=\boldsymbol{D}$, where $\boldsymbol{D}$ is a real diagonal matrix with entries $\lambda_{m}$. Recalling that the fading coefficients are complex Gaussian random variables (r.v), the $\beta_{m, n}$ 's are also complex Gaussian with mean and variance

$$
\begin{aligned}
\mu_{m, n} & =\mathrm{E}\left(\boldsymbol{\alpha}_{n}\right) \boldsymbol{V}^{H}=\sum_{k=1}^{M} \mathrm{E}\left(\alpha_{k, n}\right) v_{k, m}^{*}, \\
\sigma^{2} & =\operatorname{var}\left(\alpha_{m, n}\right) \sum_{k=1}^{M}\left|v_{k, m}\right|^{2}=\sigma_{\alpha}^{2} .
\end{aligned}
$$

Therefore, $\beta_{m, n}$ has the same variance as $\alpha_{m, n}$, but a different mean which is found as a linearly weighted sum of the mean values of $\alpha_{m, n}$. Since $\beta_{m, n}$ is nonzero mean complex Gaussian, its amplitude $\left|\beta_{m, n}\right|$ has a Rician conditional distribution with respect to $\mu_{m, n}$ which is assumed to follow Nakagami distribution given by (2) in our case. Due to the linear transformation involved, we restrict ourselves to i.i.d. Nakagami r.v.'s and consider orthogonal error events, i.e., $\boldsymbol{A}$ is a diagonal matrix.
Using the alternative form of the Gaussian $Q$-function [9], the average of the PEP over the channel statistics $\alpha_{m, n}$ (while $\mu_{m, n}$ is still treated as a constant) is given by [8]

$$
\begin{aligned}
& P\left(\boldsymbol{X}, \hat{\boldsymbol{X}} \mid \mu_{m, n}, m=1,2 \ldots M, n=1,2 \ldots N\right) \\
& =\frac{1}{\pi} \int_{0}^{\pi / 2} \prod_{n=1}^{N} \prod_{m=1}^{M} \frac{\sin ^{2} \theta}{\sin ^{2} \theta+\Omega_{m}} \exp \left(-\frac{\mu_{m, n}^{2}}{2 \sigma^{2}} \frac{\Omega_{m}}{\sin ^{2} \theta+\Omega_{m}}\right) d \theta
\end{aligned}
$$

where $\Omega_{m}=\left(E_{s} / 4 N_{0}\right) 2 \sigma^{2} \lambda_{m}$. In order to find the unconditional PEP, we still need to take an expectation of (6) with respect to $\mu_{m, n}$, i.e.,

$$
\begin{aligned}
& P(\boldsymbol{X}, \hat{\boldsymbol{X}})= \\
& \frac{1}{\pi} \int_{\theta=0}^{\pi / 2} \prod_{n=1}^{N} \prod_{m=1}^{M} \frac{\sin ^{2} \theta}{\sin ^{2} \theta+\Omega_{m}} \frac{2 \eta^{\eta}}{\Gamma(\eta) \Lambda^{\eta}} \\
& \cdot \int_{\mu_{m, n}=0}^{\infty} \mu_{m, n}^{2 \eta-1} \exp \left(-\mu_{m, n}^{2}\left(\frac{\eta}{\Lambda}+\frac{1}{2 \sigma^{2}} \frac{\Omega_{m}}{\sin ^{2} \theta+\Omega_{m}}\right)\right) d \mu_{m, n} d \theta .
\end{aligned}
$$

Exploiting i.i.d. properties of $\mu_{m, n}$ and using [10-p. 364, 3.381.4], $\int_{0}^{\infty} z^{v-1} \exp (-a z) d z=a^{-v} \Gamma(v)$, (7) results in a single finite-range integral as

$$
\begin{aligned}
P(\mathbf{X}, \hat{X})= & \frac{1}{\pi} \int_{\theta=0}^{\pi / 2} \prod_{m=1}^{M}\left(\frac{\sin ^{2} \theta}{\sin ^{2} \theta+\Omega_{m}}\right)^{N} \\
& \times\left(\frac{\sin ^{2} \theta+\Omega_{m}}{\sin ^{2} \theta+\Omega_{m}+\frac{\Lambda}{\eta} \frac{\Omega_{m}}{2 \sigma^{2}}}\right)^{\eta N} d \theta .
\end{aligned}
$$

This should be compared to the PEP expression derived in [8, eq. 12] for the R-L model in terms of an infinite summation, as shown in (9) at the bottom of the next page, where $g_{k, t}$ are coefficients computable by a recursive equation given in $[8$, Appendix $]$ and

$$
\Delta_{m}(\theta)=\frac{1}{2 \sigma^{2}} \frac{\Omega_{m}}{\sin ^{2} \theta+\Omega_{m}} \exp \left(2 m_{\mu}\right)
$$

with the parameter set $\left(\sigma, \sigma_{\mu}, m_{\mu}\right)$ specifying the degree of shadowing in the R-L model. Noting that $(\sigma, \eta, \Lambda)$ is the parameter set for the R-N channel where $\sigma$ is common for both models, the relationship between the R-L and R-N models is given as [2]

$$
m_{\mu}=\frac{1}{2}\left[\ln \left(\frac{\Lambda}{\eta}\right)+\Psi(\eta)\right] \text { and } \sigma_{\mu}^{2}=\frac{\Psi^{\prime}(\eta)}{4}
$$

based on second-order statistics matching. Here, $\Psi($.$) and \Psi^{\prime}($. represent the psi function and its derivative, respectively. Therefore, the PEP expression given in (8), i.e., derived for the R-N channel, can be used instead of (9), i.e., derived for the R-L channel, where the required equivalent channel parameter set can be simply obtained based on the transformation given in (10).

The derivation of PEP for the R-N channel also allows us to examine the design criteria for space-time codes. Setting $\theta=$ 


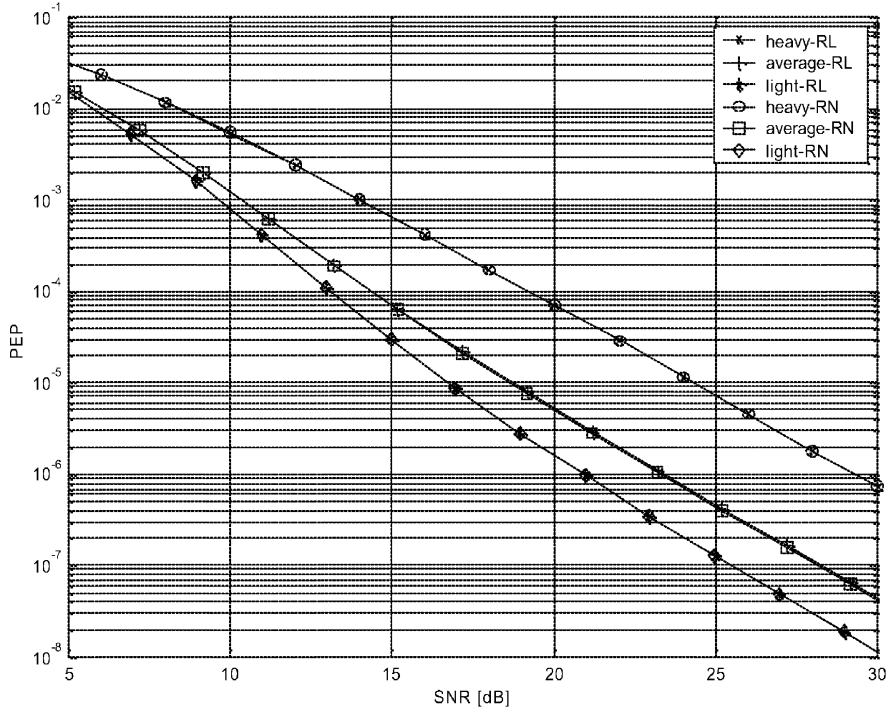

Fig. 1. Comparison of PEPs for the Rician-lognormal (RL) and the Rician-Nakagami (RN) channels.

TABLE I

RICIAN-LOGNORMAL AND RICIAN-NAKAGAMI ChanNel PARAMETER SeTS [2], [3]

\begin{tabular}{l|c|c|c|c|c}
\hline Degree of shadowing & $m_{\mu}$ & $\sigma_{\mu}$ & $\sigma^{2}$ & $\eta$ & $\Lambda$ \\
\hline Light shadowing & 0.115 & 0.115 & 0.158 & 19.4 & 1.29 \\
\hline Average shadowing & -0.115 & 0.161 & 0.126 & 10.1 & 0.835 \\
\hline Heavy shadowing & -3.914 & 0.806 & 0.063 & 0.739 & $8.97 \mathrm{e}-4$ \\
\hline
\end{tabular}

$\pi / 2$ in (8) [9, p. 506] and assuming asymptotically high SNR, an upper bound on PEP is found as

$$
\begin{aligned}
P(\boldsymbol{X}, \hat{\boldsymbol{X}}) \leq & \left(\frac{E_{s}}{4 N_{0}}\right)^{-r N}\left(\prod_{m=1}^{r} \lambda_{m}\right)^{-N} \\
& \times\left(\left(2 \sigma^{2}\right)^{1 / 1 \eta \eta}\left(1+\frac{\Lambda}{\eta 2 \sigma^{2}}\right)\right)^{-\eta r N}
\end{aligned}
$$

where $r$ denotes the rank of matrix $\boldsymbol{A}$. This result is essentially the same as [1, Eq. 10] except for the presence of the last term. Therefore, the same diversity advantage (i.e., $r N$ ) is obtained over the R-N channel as in a Rayleigh channel. The additional factor in (11) is completely characterized by the channel parameter set $(\sigma, \eta, \Lambda)$ and it can be considered as a coding gain variation due to shadowing on the LOS component, confirming also our previous observations in [8].

\section{NUMERICAL RESULTS}

We consider three different channel parameter sets for the R-L channel, corresponding to light, average and heavy shad- owing as described in [2] and [3]. These parameter sets together with the equivalent parameter sets of the R-N channel are presented in Table I. As an example for the PEP evaluation, we consider the 4-state QPSK space-time trellis code in [1, Fig. 4]. For the shortest error event of this code, the matrix $\boldsymbol{A}=(\boldsymbol{X}-\hat{\boldsymbol{X}})(\boldsymbol{X}-\hat{\boldsymbol{X}})^{H}=4 \boldsymbol{I}$ is diagonal and has two identical eigenvalues equal to 4 . Assuming one receive antenna, i.e., $N=1$, we plot PEP in Fig. 1. based on both (8) and (9) for this specific error event. For each degree of shadowing in Table I, both results demonstrate very good match, where only a negligible difference within the thickness of line is observed while (8) provides a much simpler compact form in comparison to (9).

\section{CONCLUSIONS}

We derived an exact PEP expression for space-time codes over the R-N channel. It accepts a simple single finite-range integral solution contrary to the classical R-L channel assumption, where the final form of PEP is given in terms of an infinite summation. Based on the transformation between the channel parameters of R-L and R-N channels, we furthermore demonstrated numerical results how the PEP expressions for these two channels can be related to each other.

\section{REFERENCES}

[1] V. Tarokh, N. Seshadri, and A. R. Calderbank, "Space-time codes for high data rate wireless communication: Performance criterion and code construction," IEEE Trans. Inform. Theory, vol. 44, pp. 744-765, Mar. 1998.

[2] A. Abdi, W. C. Lau, M.-S. Alouini, and M. Kaveh, "A new simple model for land mobile satellite channels: First- and second-order statistics," IEEE Trans. Wireless Commun., vol. 2, no. 3, pp. 519-528, May 2003.

[3] C. Loo, "A statistical model for a land mobile satellite link," IEEE Trans. Veh. Technol., vol. 34, pp. 122-127, Aug. 1985.

[4] M. Uysal and C. N. Georghiades, "Error performance analysis of space-time codes over Rayleigh fading channels," J. Commun. Networks, vol. 2, no. 4, pp. 351-355, Dec. 2000.

[5] G. Taricco and E. Biglieri, "Exact pairwise error probability of space-time codes," IEEE Trans. Inform. Theory, vol. 48, pp. 510-513, Feb. 2002.

[6] M. K. Simon, "Evaluation of average bit error probability for space-time coding based on a simpler exact evaluation of pairwise error probability," J. Commun. Networks, vol. 3, no. 3, Sept. 2001.

[7] - "A moment generating function (MGF)-based approach for performance evaluation of space-time code communication systems," Wireless Commun. Mobile Computing, vol. 2, no. 7, pp. 667-692, Nov. 2002.

[8] M. Uysal and C. N. Georghiades, "Effect of shadowing on the performance of space-time trellis codes," IEEE Trans. Wireless Commun., to be published.

[9] M. K. Simon and M.-S Alouini, Digital Communication Over Fading Channels: A Unified Approach to Performance Analysis. New York: Wiley, 2000.

[10] I. S. Gradshteyn and I. M. Rzyhik, Table of Integrals, Series and Products, 5th ed. San Diego, CA: Academic, 1994.

$$
P(\boldsymbol{X}, \hat{\boldsymbol{X}})=\frac{1}{\pi} \int_{\theta=0}^{\pi / 2} \prod_{m=1}^{M}\left\{\frac{\sin ^{2} \theta}{\sin ^{2} \theta+\Omega_{m}} \exp \left(-\Delta_{m}(\theta)\right)\left[1+\sum_{\substack{k=2 \\ k \text { keven }}}^{\infty} \frac{(k-1) ! !}{k !}\left(2 \sigma_{\mu}\right)^{k} \sum_{t=1}^{k} g_{k, t}\left(\Delta_{m}(\theta)\right)^{t}\right]\right\}^{N} d \theta
$$

